\title{
Highly efficient synthesis of DNA-binding polyamides using a convergent fragment-based approach
}

\author{
Andrew J. Fallows, ${ }^{1}$ Ishwar Singh, ${ }^{2 \dagger}$ Ruggero Dondi ${ }^{1 \S}$ Paul M. Cullis, ${ }^{1 *}$ Glenn A. Burley ${ }^{2 *}$ \\ ${ }^{1}$ Department of Chemistry, University of Leicester, University Road, Leicester, LE1 7RH, UK. \\ ${ }^{2}$ Department of Pure \& Applied Chemistry, University of Strathclyde, 295 Cathedral Street, Glasgow, G1 1XL, UK. \\ Supporting Information Placeholder
}

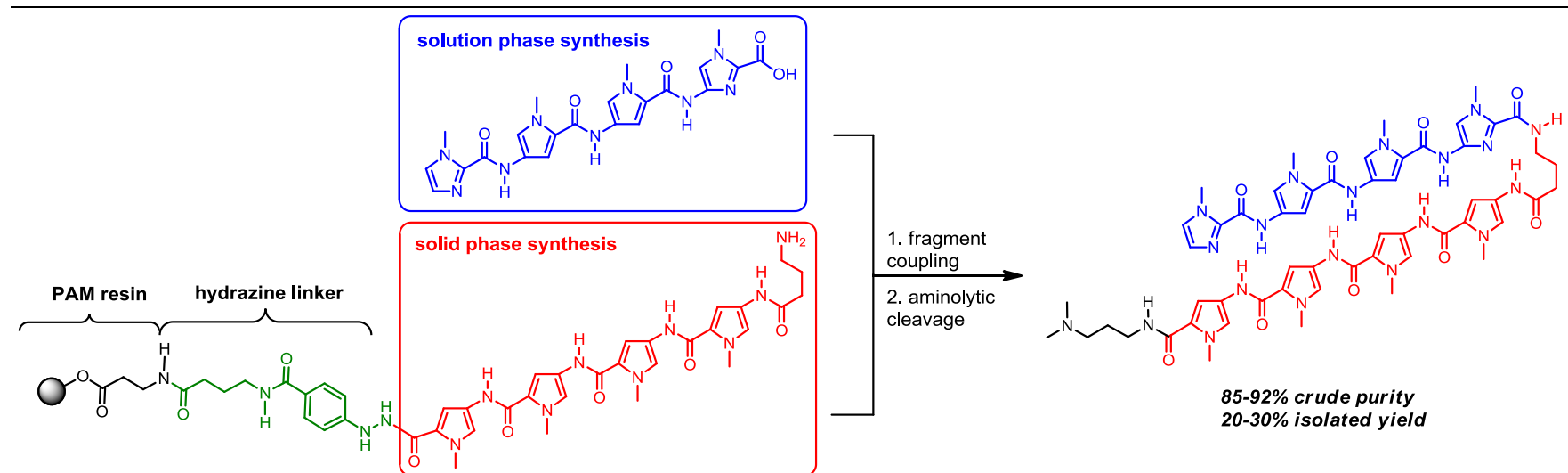

ABSTRACT: Two advances in the synthesis of hairpin pyrrole-imidazole polyamides (PAs) are described. First, the application of a convergent synthetic strategy is shown, involving the Boc-based solid phase synthesis of a $C$-terminal fragment and the solution phase synthesis of the $N$-terminal fragment. Second a new hybrid resin is developed that allows for the preparation hairpin PAs lacking a C-terminal $\beta$-alanine tail. Both methods are compatible with a range of coupling reagents and provide a facile, modular route to prepare PA libraries in high yield and crude purity.

Pyrrole-imidazole (Py-Im) polyamides (PAs) constitute a family of medium-sized molecules that bind in a sequence selective manner to double-stranded DNA (dsDNA) with nanomolar binding affinity. ${ }^{1}$ The dsDNA binding profile of PAs is highly programmable, enabling these molecules to down-regulate the expression of selective subsets of genes ${ }^{2-6}$ by targeting dsDNA sequences 7-16 base-pairs in length. ${ }^{7-9}$ Of the various PA designs that have been investigated over the past 20 years, ${ }^{1,10}$ hairpin variants have been some of the most biologically active, with examples in the literature reporting selective gene knockdown in vivo. ${ }^{411-15}$ Thus, the combined advantages of programmable perturbation of transcription factor-dsDNA interactions and cell permeability renders hairpin PAs as a potential competing gene regulatory technology to more traditional nucleic acid approaches such as RNA interference and antisense strategies.

Contingent on the wider application of PAs as biotechnological tools and potential therapeutic scaffolds is the need to prepare libraries of these compounds in which variables such as DNA binding affinity and selectivity, cell uptake, toxicity and off-target effects can be systematically assessed. Solid phase synthesis is the preferred method for the production of PAs on a milligram scale, ${ }^{16-23}$ whereas solution-phase approaches have been applied to produce a full-length hairpin PA and a cyclic version. ${ }^{24,25}$ The solid phase strategies have focused on the sequential coupling of $N$-protected monomeric (e.g., 2-5) $)^{18,21,26}$ or dimeric carboxylic acids ${ }^{22,26}$ to a primary amine immobilised on a solid support (1, Scheme 1a). The advantage of utilizing a monomeric coupling approach is the synthetic tractability of accessing the corresponding carboxylic acids such as (2) and (3). However, our experiences have shown that the coupling efficiencies are significantly lower after the $\gamma$-aminobutyric acid (GABA, 5) "hairpin turn" relative to those earlier in the synthetic cycle. We attribute this to an increase in $\pi-\pi$ interactions - either within a single PA or with adjacent PA chains - leading to reduced coupling efficiencies. $^{27-29}$ This issue is further exacerbated if the installation of $N$-methyl imidazole monomers (e.g., 3) is required late in the synthesis, as the reduced nucleophilicity of imidazole amines results in extremely poor coupling efficiencies with activated esters of (2). ${ }^{23} \mathrm{~A}$ number of approaches have been explored to overcome these synthetic challenges. ${ }^{9}$ 
Scheme 1. (a) Building blocks used in this study. (b) Coupling strategies applied in our convergent approach to PA synthesis. (c) Ligation of the two respective fragments (8/16) and (13-15) and cleavage from the resin afforded full length PAs. ${ }^{a}$ For details concerning the coupling conditions please see the Supporting Information.

(a)<smiles>NCCC(=O)O[Na]</smiles>

(1)

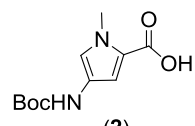

(2)

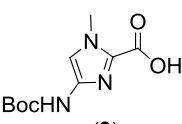

(3)

(b) hydrazine linker coupling

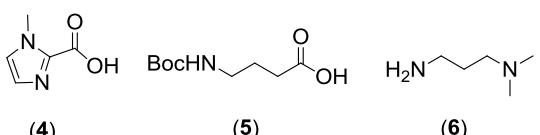

(4)
(5)

solid phase synthesis (SPS)

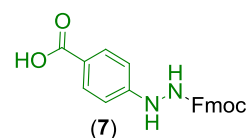

solution phase synthesis

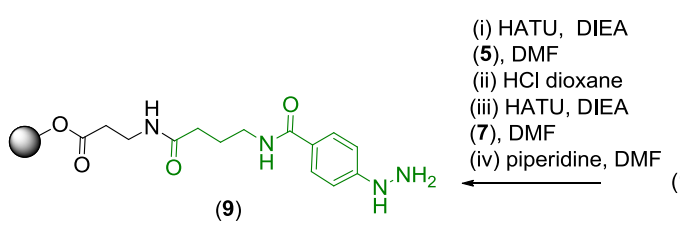

(i) HATU, DIEA

(ii) $\mathrm{HCl}$ dioxane

(7), DMF

(9)

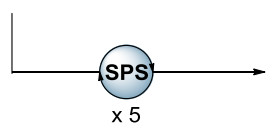

(vii) HATU, DIEA,

(2), DMF (couplings 1-4)

(5) (coupling 5)

(viii) $\mathrm{HCl}$, dioxane

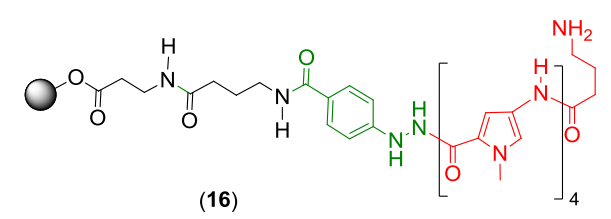

(v) HATU, DIEA

(2), DMF

(couplings 1-4)

(5) (coupling 5)

(1)

(vi) $\mathrm{HCl}$, dioxane
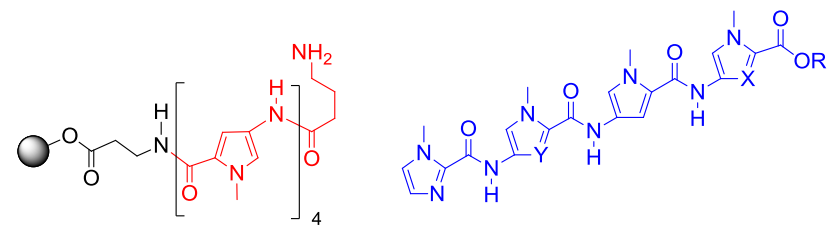

(8)

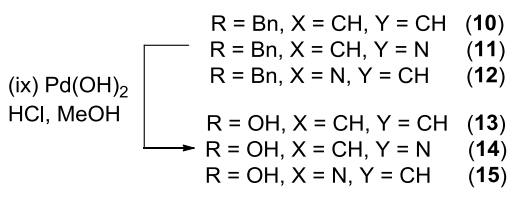

(c)

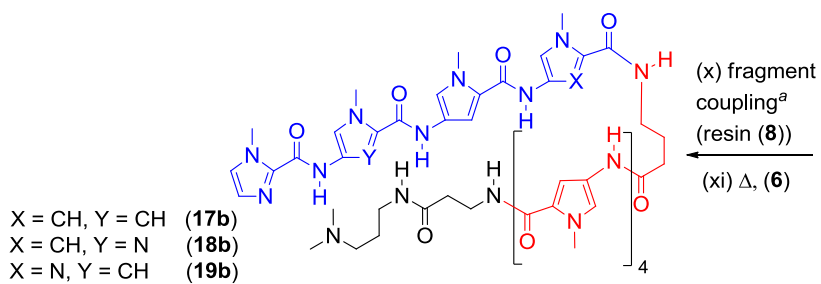

In a comprehensive study $\mathrm{Li}$ et al. reported the application of microwave irradiation to access cyclic PA libraries that are difficult to prepare by conventional methods. ${ }^{16}$ These microwave-assisted methods significantly enhance the coupling yields and reduce coupling times. These authors noted the risk of premature cleavage from the trityl resin can occur, necessitating optimisation and careful control of the reaction conditions. They also noted the difficulty in conducting immobilised imidazole-amine couplings even at elevated temperature $\left(60-80^{\circ} \mathrm{C}\right)$. Both of these issues reduce the modularity of the approach. A facile and general synthetic strategy for the synthesis of PA libraries is therefore still a key challenge.

We report here a convergent synthesis of PAs using a combined Boc-based solution/solid phase synthesis approach, leading to hairpin PA products in superior yields and purities compared to conventional sequential monomeric coupling strategies. A new hydrazine PAM hybrid resin has been developed to facilitate the synthesis of PAs that do not contain a $\beta$ Ala tail, exploiting our convergent synthetic approach. Scheme 1b-c outlines our general route to hairpin PAs (17b19b/20). We reasoned that a convergent approach involving the preparation of two requisite PA fragments of similar molecular weight would improve the yields of PA synthesis. Based on our previous experience, ${ }^{23}$ couplings of $(\mathbf{2}) /(\mathbf{3})$ to resin-immobilised amines after the turn moiety are lower yielding, requiring longer coupling times and even doubling coupling regimes using conventional coupling chemistries. Therefore an immobilised $C$-terminal fragment (e.g., 8, Scheme $1 b$ ) would be prepared by solid phase synthesis up to the turn moiety. In parallel, an $N$-terminal tetramer acid fragment (e.g., 13-15) would be prepared using a solution phase protocol to produce larger quantities $(100 \mathrm{mg})$ of the latter half of the PA. ${ }^{24} \mathrm{~A}$ final amide coupling of the two requisite frag-

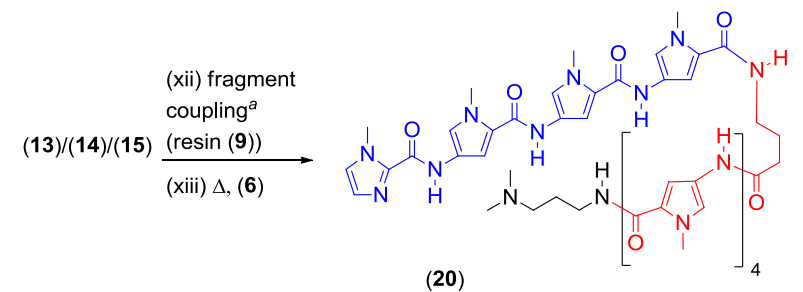

ments followed by amine cleavage would then afford the final PA products (e.g., 17b-19b or 20, Scheme 1c).

Three tetramer acids (13-15) were chosen to test this convergent synthetic strategy. These acids consist of a single $N$ methyl imidazole unit on the $N$-terminus and an $N$-methyl pyrrole carboxylic acid (13), an $N$-terminal and an internal $N$ methyl imidazole unit (14) and finally a tetramer acid with two $N$-methyl imidazole unit on the $N$ - and $C$-termini (15). The synthesis of each tetramer acid began with the formation of the benzyl esters of the carboxylic acids (2) and (3). The use of a benzyl ester monomer in the synthesis of (13-15) rather than a methyl ester used previously, ${ }^{24}$ enabled the final deprotection step to be effected via hydrogenolysis (See Supporting Information). ${ }^{30}$ This strategy avoided a saponification step required for the methyl ester, ${ }^{24}$ which in our hands proved problematic, particularly with the formation of $N$-methylimidazole acids such as (15), which are prone to decarboxylation. ${ }^{31}$

Deprotection of the benzyl esters (10-12) using Pearlman's catalyst $\left(\mathrm{Pd}(\mathrm{OH})_{2} / \mathrm{C}\right)$ under hydrogen at 50 psi produced the corresponding tetramer acids either with a terminal $N$-methyl pyrrole (13/14) or an $N$-methyl imidazole unit (15) within 5 hours and in complete conversion. Overall tetramers (13-15) were prepared using cycles of coupling and Boc-deprotection on a $100 \mathrm{mg}$ scale over 7 steps in $59-80 \%$ yield and with crude purities of the final products ranging from $89-95 \%$.

A crucial aspect in developing a facile route to hairpin PA libraries is the ability to access a variety of PA structures with combinatorial variations in the heterocyclic cores and the $C$ terminal tail unit. The heterocyclic cores define DNA sequence selectivity, ${ }^{1}$ whereas the $C$-terminal tail unit is known to influence cell uptake. ${ }^{32-34}$ By virtue of its chemical robustness and the facile conditions used to cleave PAs from the 
solid support, the commercially available Boc- $\beta$-Ala-PAM resin has been the most widely used resin type for PA synthesis. $^{26}$ The major drawback in using this resin is the installation of a $\beta$-Ala unit between the $N, N$-di-methylaminopropylamine (6) tail and the PA core. This $C$-terminal $\beta$-Ala unit is known to perturb the uptake of PAs into cells $;^{35,36}$ therefore a flexible solid phase synthetic strategy that can access PA subtypes lacking this unit is highly desirable.

In light of issues with existing methods, we set out to develop a flexible solid phase synthetic strategy that can efficiently access PA scaffolds lacking the $C$-terminal $\beta$-Ala unit. Our previous work has shown that PAs without the $C$-terminal $\beta$-Ala unit can be prepared by the sequential coupling of monomeric units (2) and (3) using a hydrazinobenzoyl resin. ${ }^{23}$ Furthermore, powerful condensing agents such as BTC [bis(trichloromethyl) carbonate] could be used to couple challenging $N$-methyl pyrrole units (2) to resin-bond $N$-methyl imidazole amines in almost quantitative yield. ${ }^{23}$ Unfortunately we have experienced batch-to-batch variability with commercially-available resin types which reduced the yield and purity of final PA products. Therefore, we developed a new hybrid resin $(9$, Scheme $1 b)$, which combined the robustness of the Boc- $\beta$-Ala-PAM resin used extensively in PA synthesis ${ }^{26}$ with the flexibility of the hydrazinobenzoyl resin. ${ }^{23}$ The hybrid resin (9) was prepared by a HATU-mediated coupling of a GABA unit (5) to (1), followed by a Boc-deprotection and a HATU-mediated coupling of the hydrazine acid (7) and a final Fmoc deprotection. A representative $C$-terminal tetramer (16) immobilised on a solid support was then synthesised on this resin by sequential monomeric coupling according to previously reported coupling protocols. ${ }^{23,26}$

With the respective $N$-terminal (13-15) and $C$-terminal (8) PA fragments in hand, the yield and crude purity of a suite of three PAs was then explored using our convergent fragment strategy and the yields compared to a PA library prepared via sequential monomeric couplings (Table 1). The final isolated yields of PAs derived from our fragment strategy (17b-19b) were approximately 3-times higher than PAs prepared by traditional monomeric coupling strategies (17a-19a,). Furthermore, the crude purity of all PAs prepared by this method (17b-19b) was $85-90 \%$ (as determined by RP-HPLC @ 310 $\mathrm{nm})$, compared to $68-78 \%$ for PAs (17a-19a) prepared by traditional methods (Fig. 1).

A suite of four 6-ring hairpin PAs (21-24) were then prepared using an array of coupling agents in order to ascertain the flexibility of our new hybrid resin. Our hybrid resin demonstrated excellent compatibility with conventional coupling agents such as HATU, DCC/HOAt and BTC using monomers (2), (3) and (5) to produce PAs (21-24). These PA sequences include a challenging coupling in the 6 position between a resin-bound $N$-methylimidazole amine with (2) using BTC as the coupling agent to produce PA (23) in $12 \%$ yield, whereas the PA (24) incorporating the Fmoc-D-Dab(Boc)-OH turn was prepared in $13 \%$ yield. Crucially, PA fragments prepared on our hybrid resin afforded PAs (PAs, 21-24) in yields and crude purities comparable to that of PA prepared on a conventional $\beta$-Ala-PAM resin (PAs 17a-19a Table 1).

An unexpected result that we observed during the course of this study was the facile conditions required to cleave PAs (21-24) derived from the hybrid linker (9). Traditional cleavage conditions of aryl hydrazine resins require oxidation of the
Table 1: Crude purity and isolated yields of PAs prepared through standard protocols ${ }^{1}$ and our convergent strategy.

\begin{tabular}{|c|c|c|c|}
\hline $\begin{array}{l}\text { compound } \\
\text { number }\end{array}$ & $\diamond=\beta-\mathrm{Ala} \bigcirc=\mathrm{Py} \quad=\mathrm{Im}$ & $\begin{array}{l}\text { purity } \\
(\%)\end{array}$ & $\begin{array}{l}\text { yield } \\
(\%)\end{array}$ \\
\hline $17 \mathrm{a}^{\mathrm{a}}$ & & 68 & 12 \\
\hline $18 \mathrm{a}^{\mathrm{a}}$ & & 76 & 10 \\
\hline $19 \mathrm{a}^{\mathrm{a}}$ & & 78 & 11 \\
\hline $21^{\mathrm{a}}$ & & 68 & 11 \\
\hline $22^{\mathrm{a}}$ & & 70 & 11 \\
\hline $23^{\mathrm{a}}$ & & 74 & 12 \\
\hline $24^{\mathrm{a}}$ & & 79 & 13 \\
\hline $17 b^{b}$ & & 90 & 30 \\
\hline $18 b^{b}$ & & 88 & 30 \\
\hline $19 b^{b}$ & & 85 & 28 \\
\hline $20^{\mathrm{b}}$ & & 87 & 20 \\
\hline
\end{tabular}

${ }^{a}$ PAs prepared by monomeric couplings. ${ }^{b}$ PAs prepared by a fragment-based approach.
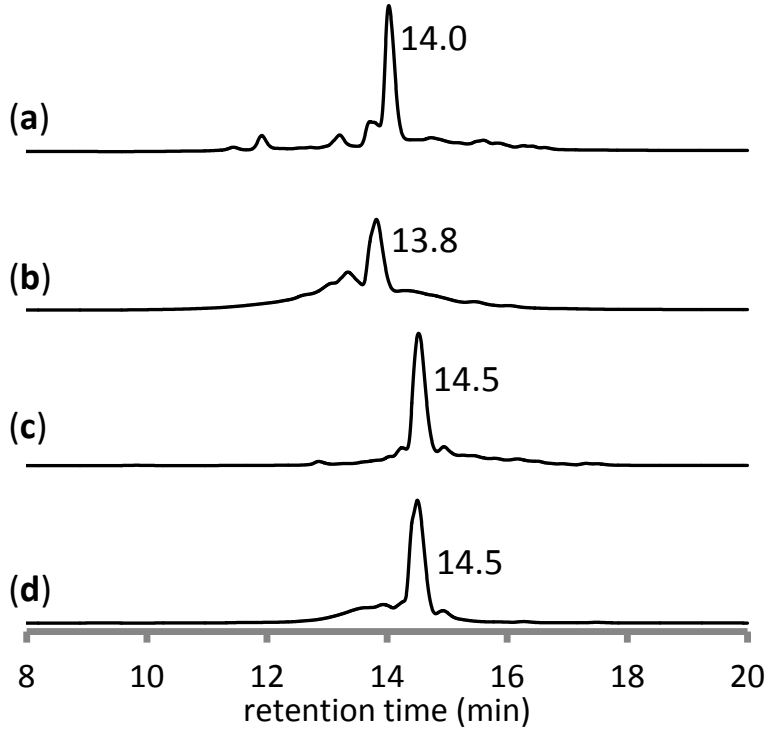

Fig. 1: Crude HPLC profiles of (a) Compound (17a). Crude purity was 69\%. (b) Compound (21). Crude purity was $68 \%$. (c) Compound (17b). Crude purity was $90 \%$. (d) Compound (20). Crude purity was $87 \%$.

hydrazide by $\mathrm{N}$-bromosuccinimide or atmospheric $\mathrm{O}_{2}$ in the presence of $\mathrm{Cu}(\mathrm{II})$ salts to form a transient diazene species. ${ }^{23,37}$ We observed that initial activation/oxidation of the resin was not required and aminolysis $\left(55^{\circ} \mathrm{C}\right.$ for 16 hours) of resin- 
bound PAs with amine (6) under air resulted in facile cleavage to form PAs (21-24) in high yield and crude purity.

We therefore conclude that the isolated yield and purity of PAs can be improved by the application of a fragment-based synthetic approach. This correlates to an average $94 \%$ yield per reaction cycle (i.e. coupling and Boc-deprotection) for an eight ring PA prepared by our fragment-based approach compared to an average $89 \%$ yield per cycle when a monomeric coupling approach is used. Although a 5\% increase in yield per reaction cycle appears modest, this constitutes a significant enhancement in overall isolated yield and purity. We reason that the enhancement of the isolated yield of PAs is likely due to the reduction of the number of couplings undertaken on the solid support; a phenomenon observed when a fragment-based approach was applied to peptides and small molecules. ${ }^{38,39}$

Second, the coupling of hydrazine acid (7) to a $\beta$-AlaPAM resin via a GABA linker enables the facile preparation of PAs lacking the $C$-terminal $\beta$-Ala unit. This simple modification increases the flexibility of the synthesis of PAs and opens up opportunities for the direct installation of other functional groups on the $C$-terminus. ${ }^{33,35,36}$ Efforts are now underway to apply this methodology to prepare PA libraries incorporating structural variations at key sites of the PA scaffold in order to enhance the biological activity of these compounds in cells.

\section{Supporting Information}

Synthesis and analytical characterization of PAs can be found in the Supporting Information. This material is available free of charge via the Internet at http://pubs.acs.org.

\section{AUTHOR INFORMATION}

\section{Corresponding Authors}

* pmc@le.ac.uk; glenn.burley@strath.ac.uk

\section{Present Addresses}

${ }^{\dagger}$ School of Pharmacy, University of Lincoln, Brayford Pool, Lincoln, LN6 7TS, UK.

${ }^{\S}$ Department of Pharmacy and Pharmacology, University of Bath, Claverton Down, Bath, BA2 7AY, UK.

\section{ACKNOWLEDGMENTS}

G.A.B. thanks the EPSRC for funding (ARF, EP/E055095/1 and $\mathrm{EP} / \mathrm{H} 02915 \mathrm{X} / 1)$. A.J.F. thanks the EPSRC and the University of Leicester for a postgraduate studentship.

\section{REFERENCES}

(1) Dervan, P. B.; Edelson, B. S. Curr. Opin. Struct. Biol. 2003, 13, 284.

(2) Raskatov, J. A.; Meier, J. L.; Puckett, J. W.; Yang, F.; Ramakrishnan, P.; Dervan, P. B. Proc. Natl. Acad. Sci. U.S.A. 2012 109, 1023.

(3) Muzikar, K. A.; Nickols, N. G.; Dervan, P. B. Proc. Natl. Acad. Sci. U.S.A. 2009, 106, 16598.

(4) Warren, C. L.; Kratochvil, N. C. S.; Hauschild, K. E.; Foister, S.; Brezinski, M. L.; Dervan, P. B.; Phillips, G. N.; Ansari, A. Z. Proc. Natl. Acad. Sci. U.S.A. 2006, 103, 867.

(5) Kashiwazaki, G.; Bando, T.; Yoshidome, T.; Masui, S.; Takagaki, T.; Hashiya, K.; Pandian, G. N.; Yasuoka, J.; Akiyoshi, K.; Sugiyama, H. J. Med. Chem. 2012, 55, 2057.

(6) Pandian, G. N.; Shinohara, K.-i.; Ohtsuki, A.; Nakano, Y.; Masafumi, M.; Bando, T.; Nagase, H.; Yamada, Y.; Watanabe, A.;
Terada, N.; Sato, S.; Morinaga, H.; Sugiyama, H. ChemBioChem 2011, 12, 2822.

(7) Trauger, J. W.; Baird, E. E.; Dervan, P. B. J. Am. Chem. Soc. 1998, 120, 3534 .

(8) Yamamoto, M.; Bando, T.; Kawamoto, Y.; Taylor, R. D.; Hashiya, K.; Sugiyama, H. Bioconjugate Chem. 2014, 25, 552.

(9) Kawamoto, Y.; Bando, T.; Kamada, F.; Li, Y.; Hashiya, K.; Maeshima, K.; Sugiyama, H. J. Am. Chem. Soc. 2013, 135, 16468.

(10) Bando, T.; Sugiyama, H. Acc. Chem. Res. 2006, 39, 935.

(11) Yang, F.; Nickols, N. G.; Li, B. C.; Marinov, G. K.; Said, J. W.; Dervan, P. B. Proc. Natl. Acad. Sci. U.S.A. 2013, 110, 1863.

(12) Raskatov, J. A.; Nickols, N. G.; Hargrove, A. E.; Marinov, G. K.; Wold, B.; Dervan, P. B. Proc. Natl. Acad. Sci. U.S.A. 2012, 109, 16041.

(13) Yao, E.-H.; Fukuda, N.; Ueno, T.; Matsuda, H.; Nagase, H.; Matsumoto, Y.; Sugiyama, H.; Matsumoto, K. Cardiovasc. Res. 2009, $81,797$.

(14) Ueno, T.; Fukuda, N.; Tsunemi, A.; Yao, E.-H.; Matsuda, H.; Tahira, K.; Matsumoto, T.; Matsumoto, K.; Matsumoto, Y.; Nagase, H.; Sugiyama, H.; Sawamura, T. J. Hypertension 2009, 27, 508.

(15) Wang, X.; Nagase, H.; Watanabe, T.; Nobusue, H.; Suzuki, T.; Asami, Y.; Shinojima, Y.; Kawashima, H.; Takagi, K.; Mishra, R.; Igarashi, J.; Kimura, M.; Takayama, T.; Fukuda, N.; Sugiyama, H. Cancer Sci. 2010, 101, 759.

(16) Li, B. C.; Montgomery, D. C.; Puckett, J. W.; Dervan, P. B. J. Org. Chem. 2013, 78, 124

(17) Wurtz, N. R.; Turner, J. M.; Baird, E. E.; Dervan, P. B. Org. Lett. 2001, 3, 1201.

(18) Krutzik, P. O.; Chamberlin, A. R. Bioorg. Med. Chem. Lett. 2002, 12, 2129.

(19) Choi, J. S.; Lee, Y. J.; Kim, E.; Jeong, N.; Yu, H.; Han, H. Tetrahedron Lett. 2003, 44, 1607.

(20) Bando, T.; Fujimoto, J.; Minoshima, M.; Shinohara, K. I.; Sasaki, S.; Kashiwazaki, G.; Mizumura, M.; Sugiyama, H. Bioorg. Med. Chem. 2007, 15, 6937.

(21) Puckett, J. W.; Green, J. T.; Dervan, P. B. Org. Lett. 2012, 14, 2774.

(22) Wetzler, M.; Wemmer, D. E. Org. Lett. 2010, 12, 3488.

(23) Su, W.; Gray, S. J.; Dondi, R.; Burley, G. A. Org. Lett. 2009, 11, 3910 .

(24) Chenoweth, D. M.; Harki, D. A.; Dervan, P. B. J. Am. Chem. Soc. 2009, 131, 7175.

(25) Chenoweth, D. M.; Harki, D. A.; Phillips, J. W.; Dose, C.; Dervan, P. B. J. Am. Chem. Soc. 2009, 131, 7182.

(26) Baird, E. E.; Dervan, P. B. J. Am. Chem. Soc. 1996, 118, 6141.

(27) Matmour, R.; De Cat, I.; George, S. J.; Adriaens, W.; Leclère, P.; Bomans, P. H. H.; Sommerdijk, N. A. J. M.; Gielen, J. C.; Christianen, P. C. M.; Heldens, J. T.; van Hest, J. C. M.; Löwik, D. W. P. M.; De Feyter, S.; Meijer, E. W.; Schenning, A. P. H. J. J. Am. Chem. Soc. 2008, 130, 14576.

(28) Diegelmann, S. R.; Gorham, J. M.; Tovar, J. D. J. Am. Chem. Soc. 2008, 130, 13840.

(29) Vadehra, G. S.; Wall, B. D.; Diegelmann, S. R.; Tovar, J. D. Chem. Commun. 2010, 46, 3947.

(30) Grehn, L.; Ragnarsson, U. J. Org. Chem. 1981, 46, 3492.

(31) Heckel, A.; Dervan, P. B. Chem. Eur. J. 2003, 9, 3353.

(32) Jacobs, C. S.; Dervan, P. B. J. Med. Chem. 2009, 52, 7380.

(33) Liu, B.; Kodadek, T. J. Med. Chem. 2009, 52, 4604.

(34) Nishijima, S.; Shinohara, K.-i.; Bando, T.; Minoshima, M.; Kashiwazaki, G.; Sugiyama, H. Bioorg. Med. Chem. 2010, 18, 978.

(35) Edelson, B. S.; Best, T. P.; Olenyuk, B.; Nickols, N. G.; Doss, R. M.; Foister, S.; Heckel, A.; Dervan, P. B. Nucleic Acids Res. 2004, 32, 2802.

(36) Best, T. P.; Edelson, B. S.; Nickols, N. G.; Dervan, P. B. Proc. Natl. Acad. Sci. U.S.A. 2003, 100, 12063.

(37) Góngora-Benítez, M.; Tulla-Puche, J.; Albericio, F. ACS Combin. Sci. 2013, 15, 217.

(38) Dalcol, I.; Rabanal, F.; Ludevid, M.-D.; Albericio, F.; Giralt, E. J. Org. Chem. 1995, 60, 7575 .

(39) Chen, C.; Li, X.; Neumann, C. S.; Lo, M. M. C.; Schreiber, S. L. Angew. Chemie Int. Ed. 2005, 44, 2249. 

